\title{
Contrastive Domain Adaptation
}

\author{
Mamatha Thota \\ School of Computer Science \\ University of Lincoln \\ Lincoln, LN6 7TS, UK \\ mthota@lincoln.ac.uk
}

\begin{abstract}
Recently, contrastive self-supervised learning has become a key component for learning visual representations across many computer vision tasks and benchmarks. However, contrastive learning in the context of domain adaptation remains largely underexplored. In this paper, we propose to extend contrastive learning to a new domain adaptation setting, a particular situation occurring where the similarity is learned and deployed on samples following different probability distributions without access to labels. Contrastive learning learns by comparing and contrasting positive and negative pairs of samples in an unsupervised setting without access to source and target labels. We have developed a variation of a recently proposed contrastive learning framework that helps tackle the domain adaptation problem, further identifying and removing possible negatives similar to the anchor to mitigate the effects of false negatives. Extensive experiments demonstrate that the proposed method adapts well, and improves the performance on the downstream domain adaptation task.
\end{abstract}

\section{Introduction}

Over the last few years, Deep Learning (DL) [22] has been successfully applied across numerous applications and domains due to the availability of large amounts of labeled data, such as computer vision and image processing [34, 42, 37, 8], signal processing [2, 33, 15], autonomous driving [26, 41, 11], agri-food technologies [1, 20], medical imaging [19, 25], etc. Most of the applications of DL techniques, such as the aforementioned ones, refer to supervised learning, it requires manually labeling a dataset, which is a very time consuming, cumbersome and expensive process that has led to the widespread use of certain datasets, e.g. ImageNet, for model pre-training. On the other hand, unlabeled data is being generated in abundance through sensor networks, vision systems, satellites, etc. One way to make use of this huge amount of unlabeled data is to get super-

\author{
Georgios Leontidis \\ Department of Computing Science \\ University of Aberdeen \\ Aberdeen, AB24 3UE, UK \\ georgios.leontidisdabdn.ac.uk
}

vision from the data itself. Since unlabeled data are largely available and are less prone to labeling bias issues, they tend to provide visual information independent from specific domain styles.

Nowadays, self-supervised visual representation learning has been largely closing the gap with, in some cases, even surpassing supervised learning methods. One of the most prominent self-supervised visual representation learning techniques that has been gaining popularity is contrastive learning, which aims to learn an embedding space by contrasting semantically positive and negative pairs of samples $[4,5,13]$.

However, whether these self-supervised visual representation learning techniques can be efficiently applied for domain adaptation has not yet been satisfactorily explored. When, one applies a well performing model learned from a source training set to a different but related target test set, generally the assumption is that both these sets of data are drawn from the same distributions. When this assumption is violated, the DL model trained on the source domain data will not generalize well on the target domain, due to the distribution differences between the source and the target domains known as domain shift. Learning a discriminative model in the presence of domain shift between source and target datasets is known as Domain Adaptation.

Existing domain adaptation methods rely on rich prior knowledge about the source data labels, which greatly limits their application, as explained above. This paper introduces a contrastive learning based domain adaptation approach that requires no prior knowledge of the label sets. The assumption is that both the source and target datasets share the same labels, but only the marginal probability distributions differ.

One of the fundamental problems with contrastive selfsupervised learning is the presence of potential false negatives that need to be identified and eliminated; but without labels, this problem is rather difficult to solve. Some notable work related to this area has been proposed in [17] and [35], where both methods focused on mining hard negatives; [16] 
developed a method for false negative elimination and false negative attraction and [7] proposed a method to correct the sampling bias of negative samples.

Over the past few years, ImageNet pre-training has become a standard practice, but using contrastive learning has demonstrated a competitive performance without access to labeled data by training the encoder using the input data itself. In this paper, we extend contrastive learning also referred as unsupervised representation learning without access to labeled data or pretrained imagenet weights, where we leverage the vast amount of unlabeled source and target data to train an encoder using random initialized parameters to the domain adaptation setting, a particular situation occurring where the similarity is learned and deployed on samples following different probability distributions. We also present an approach to address one of the fundamental problems of contrastive representation learning, i.e. identifying and removing the potential false negatives. We performed various experiments and tested our proposed model and its variants on several benchmarks that focus on the downstream domain adaptation task, demonstrating a competitive performance against baseline methods, albeit not using any source or target labeled data.

The rest of the paper is laid out as follows: Section 2 presents the related work in self-supervised contrastive representation learning and domain adaptation methods. Section 3 describes our proposed approach, Section 4 presents the datasets and experimental results on domain adaptation after applying our model, and finally, Section 5 summarizes our work and future directions.

\subsection{Contributions}

The main contributions of this work can be summarised as follows:

- We explore contrastive learning in the context of Domain Adaptation, attempting to maximize generalization between source and target domains with different distributions.

- We propose a Domain Adaptation approach that does not make use of any labeled data or involves imagenet pretraining.

- We incorporate false negative elimination to the domain adaptation setting, resulting in improved accuracy and without incurring any additional computational overhead.

- We extend our domain adaptation framework and perform various experiments to learn from more than two views.

\section{Related Work}

Domain Adaptation: Domain adaptation is a special case of transfer learning where the goal is to learn a discriminative model in the presence of domain shift between source and target datasets. Various methods have been introduced to minimize the domain discrepancy in order to learn domain-invariant features. Some involve adversarial methods like DANN [10], ADDA[39] that help align source and target distributions. Other methods propose aligning distributions through minimizing divergence using popular methods like maximum mean discrepancy $[12,28,29]$, correlation alignment [36, 3], and the Wasserstein metric $[6,24]$. MMD was first introduced for the two-sample tests of the hypothesis that two distributions are equal based on observed samples from the two distributions [12], and this is currently the most widely used metric to measure the distance between two feature distributions. The Deep Domain Confusion Network proposed by Tzeng et al.[40] learns both semantically meaningful and domain invariant representations, while Long et al. proposed DAN [28] and JAN [29] which both perform domain matching via multi-kernel MMD (MK-MMD) or a joint MMD (J-MMD) criteria in multiple domain-specific layers across domains.

Contrastive Learning: Recently, contrastive learning has achieved state-of-the-art performance in representation learning, leading to state-of-the-art results in computer vision. The aim is to learn an embedding space where positive pairs are pulled together, whilst negative pairs are pushed away from each other. Positive pairs are drawn by pairing the augmentations of the same image, whereas the negative pairs are drawn from different images. Existing contrastive learning methods have different strategies to generate positive and negative samples. Wu et al.[43] maintains all the sample representations of the images in a memory bank, MoCo [13] maintains an on-the-fly momentum encoder along with a limited queue of previous samples, Tian et al.[38] uses all the generated multi view samples with the mini-batch approach, whereas both SimClr V1 [4] and SimClr V2 [5] use momentum encoder and utilize all the generated sample representations within the mini batch. The above methods can provide a pretrained network for a downstream task, but do not consider domain shift if they are applied directly. However, our approach aims to learn representations that are generalizable without any need of labeled data. Recently, contrastive learning was applied in Unsupervised Domain Adaptation setting [18, 32, 21], where models have access to the source labels and/or used models pretrained on imagenet as their backbone network. In comparison, our work is based on contrastive learning, which is also referred to as unsupervised representation learning, without having access to labeled data or pretrained imagenet parameters, but instead leveraging the vast amount of unlabeled source and target 


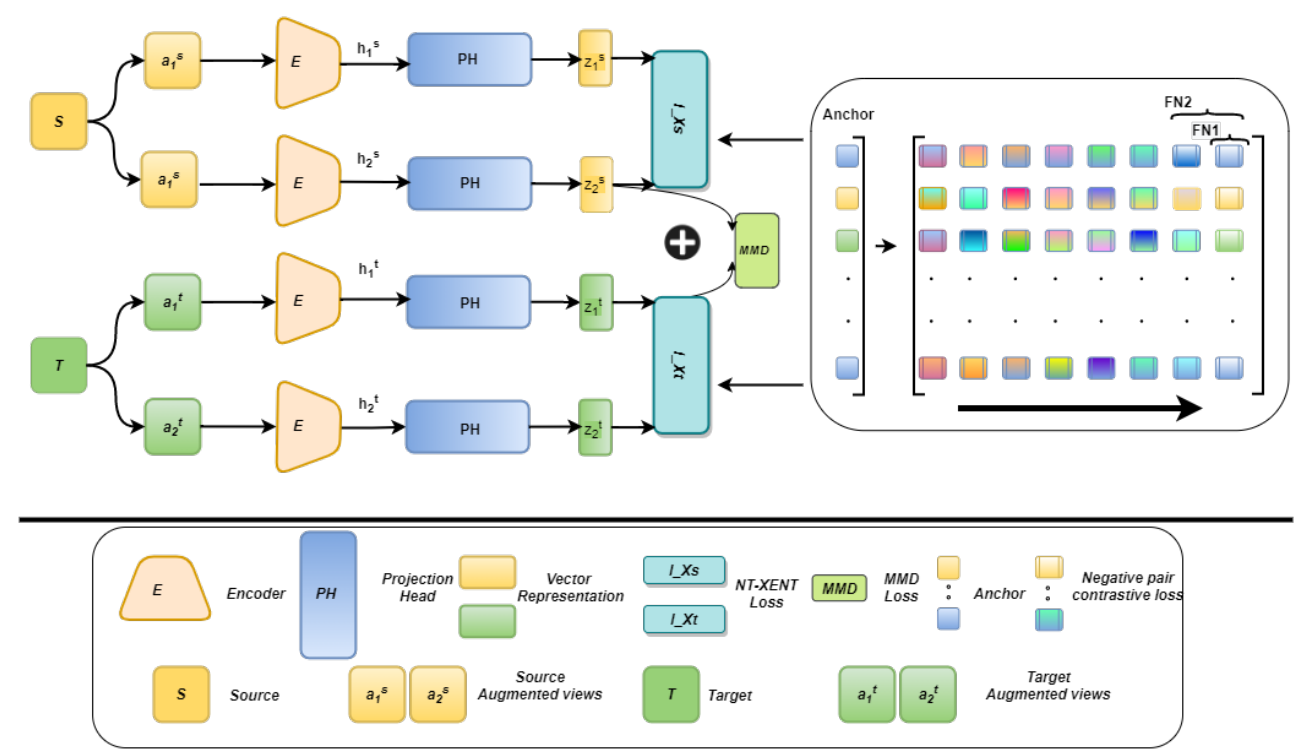

Figure 1: Overview of our proposed Contrastive Domain Adaptation model. Image on the Left, shows the pipeline of our model and image on the Right shows the loss function.

data to train a encoder from random initialized parameters.

Removal of false negatives: As the name suggests, contrastive learning methods learn by contrasting semantically similar and dissimilar pairs of samples. They rely on the number of negative samples for generating good quality representations and favor large batch size. As we do not have access to labels, when an anchor image is paired with the negative samples to form a negative pair, there is a probability that these images could share the same class, in which case the contribution towards the contrastive loss becomes minimal, limiting the ability of the model to converge quickly. These false negatives remain a fundamental problem in contrastive learning methodology, but relatively limited work has been done in this area thus far.

Most existing methods focus on mining hard negatives; [17] developed hard negative mixing to synthesize hard negatives on the fly in the embedding space, [35] developed new sampling methods for selecting hard negative samples where the user can control the hardness, [16] proposed an approach for false negative elimination and false negative attraction and [7] developed a debiased contrastive objective that corrects for the sampling bias of negative samples. [16] use additional support views and aggregation as part of their elimination and attraction strategy. Regarding our proposed approached, and inspired by [16], we have simplified and only applied the false elimination part to the domain adaptation framework. Instead of using additional support views, we compute the similarity loss between the anchor and the negatives in the mini-batch, we then sort the corre- sponding negative pair similarity losses for each anchor and remove the negative pair similar to the anchor. For each anchor in the mini-batch, we remove the exact same number of negative pairs; for example, in FNR_1 we remove one potential false negative from a total of 1023 negative samples with a batch size of 512 , totalling 512 total potential false negatives for all the anchor images in the mini-batch of 512 .

\section{Method}

\subsection{Model Overview}

Contrastive Domain Adaptation (CDA): We explore a new domain adaptation setting in a fully self-supervised fashion without any labeled data being used from either source or target domain. In the normal UDA setting, one has access to the source domain labels, but our goal is to train a model using unlabeled data sources in order to generalize visual features in both source and target domains. The aim is to obtain pre-trained weights that are robust to domain-shift and generalizable to the downstream domain adaptation task. Our model uses unlabeled source and target datasets in an attempt to learn and solve the adaptation between domains.

Inspired by the recent successes of learning from unlabeled data, the proposed learning framework is based on SimClr [4] for the domain adaptation setting, where data from unlabeled source and target domains is used in a taskagnostic way. SimClr [4] method learns visual similarity 


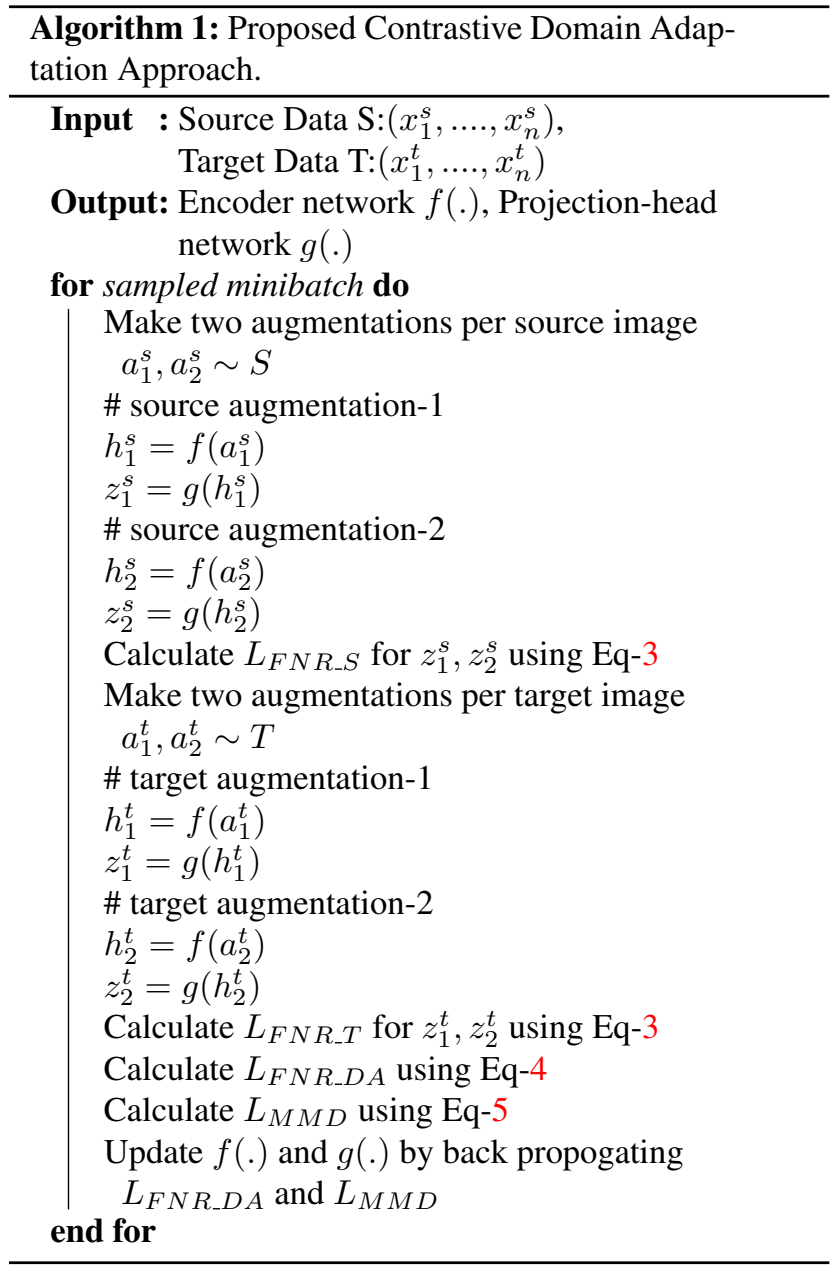

where a model pulls together visually similar-looking images, while pushing away dissimilar-looking images. However, in domain adaptation, the same class images may look very different due to domain gap, so that learning visual similarity alone does not ensure semantic similarity and domain-invariance between domains. Therefore, via using CDA, we aim to learn general visual class-discriminative and domain-invariant features from both the domains via unsupervised pretraining. We introduce each specific component in detail below, which is also illustrated in Figure-1 and Figure-2 for four views.

From randomly sampled mini-batch of images $N$, we augment each image $S$ twice creating two views of same anchor image $s_{i}$ and $s_{j}$. We use a base encoder (Resnet50 architecture [14]) that is trained from scratch to encode augmented images in order to generate representations $h s_{i}$ and $h s_{j}$. These representations are then inputted into a nonlinear MLP with two hidden layers to get the projected vector representations $z s_{i}$ and $z s_{j}$. We find that this MLP projection benefits our model by compressing our images into a latent space representation, enabling the model to learn the high-level features of the images. We apply contrastive loss on the vector representations using the NT-Xent loss [4] that has been modified to identify and eliminate false negatives, thus resulting in improved accuracy, details of which are discussed in section 4.2. We also introduce MMD to measure domain discrepancy in feature space in order to minimize domain shift, details of which are discussed later in this section as well. The overarching aim is to obtain the pretrained weights that are robust to domain-shift and efficiently generalizable. In the later stage, we perform linear evaluation using the encoder whilst entirely discarding the MLP projection head after pretraining.

\subsection{Contrastive Loss for Domain Adaptation}

The goal of contrastive learning is to maximize the similarities between positive pairs and minimize the similarities of negative ones. We randomly sample mini batch of $N$ images, each anchor image $x$ is augmented twice creating two views of the same sample $x_{i}$ and $x_{j}$, resulting in $2 N$ images. We do not explicitly sample the negative pairs, we instead follow [4], and treat other 2(N-1) augmented image samples as negative pairs. The contrastive loss is defined as follows:

$$
L_{C O N T}=-\log \frac{\exp \left(\operatorname{sim}\left(z_{i}, z_{j}\right) / T\right)}{\sum_{k=1}^{2 N} 1_{(k \neq i)} \operatorname{sim}\left(z_{i}, z_{k}\right) / T}
$$

where $\operatorname{sim}(u, v)$ is a cosine similarity function $u^{T} v /\|u\|\|v\|$ and $T$ is a temperature parameter.

However, If we use the above contrastive loss as used in a domain adaptation scenario, considering that the minibatch contains image samples from both domains, it may treat all other samples as negatives against the anchor image even though they may belong to the same class, without distinguishing domains; this could further widen the distance between them due to the difference in the domain specific visual characteristics, and therefore be unable to learn domain invariance. In order to overcome these problems, we propose to perform contrastive learning in the source and target domain independently by randomly sampling instances from both domains. Finally, our contrastive loss for DA is defined as follows:

$$
L_{C O N T_{-} D A}=L_{C O N T_{-} S}+L_{C O N T_{-} T}
$$

where $L_{C O N_{-} S}$ and $L_{C O N_{-} T}$ are source contrastive loss and target contrastive loss

\subsection{Removal of False Negatives}

Unsupervised contrastive representation learning methods aim to learn by contrasting semantically positive and negative pairs of samples. As we do not have access to the true labels in this type of setting, positive pairs are drawn 


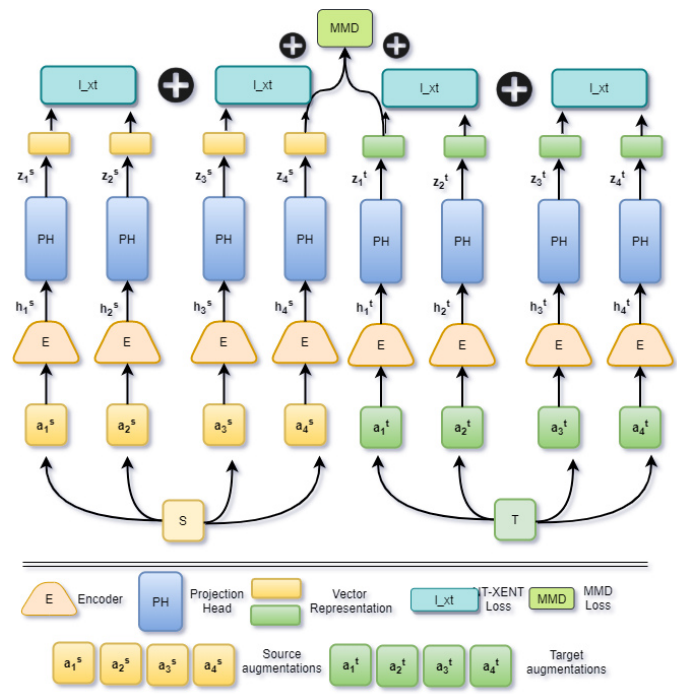

Figure 2: Overview of CDA with four views

by pairing the augmentations of the same image, whereas the negative pairs are drawn from different images within the same batch. For instance, for a batch of $N$ images, augmented images form $N$ positive pairs for a total of $2 N$ images and $2 N-1$ negative pairs. From those $2 N-1$, there could be images which are similar to the anchor, hence treated as false negative.

During training, an augmented anchor image is compared against the negative samples to contribute towards a contrastive loss, and as a result, there is a possibility that some of these pairs may have the same semantic information (label) as that of the anchor, and therefore can be treated as false negatives. But in cases where the original image sample and a negative image sample share the same class, the contribution towards the contrastive loss becomes minimal, limiting the ability of the model to converge quickly, as the presence of these false negatives can discard semantic information leading to significant performance drop. We therefore identify and remove the negatives that are similar to the anchor in order to improve the performance of the contrastive learning.

After removing the false negatives, the contrastive loss can be defined as follows:

$$
L_{F N R}=-\log \frac{\exp \left(\operatorname{sim}\left(z_{i}, z_{j}\right) / T\right)}{\sum_{k=1}^{2 N} 1_{\left(k \neq i, k \neq S_{i}\right)} \operatorname{sim}\left(z_{i}, z_{k}\right) / T}
$$

where $S i$ is the set of the negative pair that are similar to the anchor $i$.

However, If we use the above loss in the domain adaptation scenario - similar to the contrastive loss - considering that the mini-batch contains image samples from both domains, it may treat all other samples as negatives against the anchor image even though they may belong to the same class without distinguishing domains, further widening the distance between them due to the difference in the domain specific visual characteristics, hence being unable to learn domain invariance. In order to overcome these problems, we propose to use FNR loss in the source and target domain independently by randomly sampling instances from source and target domain. Finally, our joint FNR loss for DA is defined as follows:

$$
L_{F N R \_D A}=L_{F N R \_S}+L_{F N R \_T}
$$

where $L_{F N R_{-} S}$ and $L_{F N R_{-} T}$ are source contrastive loss and target contrastive loss

\subsection{Revisiting Maximum Mean Discrepancy}

MMD defines the distance between the two distributions with their mean embeddings in the Reproducing Kernel Hilbert Space (RKHS). MMD is a two sample kernel test to determine whether to accept or reject the null hypothesis $p=q$ [12], where $p$ and $q$ are source and target domain probability distributions. MMD is motivated by the fact that if two distributions are identical, all of their statistics should be the same. The empirical estimate of the squared MMD using two datasets is computed by the following equation:

$$
\begin{gathered}
L_{M M D}=\left\|\frac{1}{N} \sum_{i=1}^{N} \phi\left(x_{i}^{s}\right)-\frac{1}{M} \sum_{j=1}^{M} \phi\left(x_{j}^{t}\right)\right\|_{H}^{2} \\
=\frac{1}{N^{2}} \sum_{i=1}^{N} \sum_{i^{\prime}=1}^{N} k\left(x_{i}^{s}, x_{i^{\prime}}^{s}\right)-\frac{2}{N M} \sum_{i=1}^{N} \sum_{j=1}^{M} k\left(x_{i}^{s}, x_{j}^{t}\right) \\
+\frac{1}{M^{2}} \sum_{j=1}^{M} \sum_{j=1}^{M} k\left(x_{j}^{t}, x_{j^{\prime}}^{t}\right)
\end{gathered}
$$

where $\phi($.$) is the mapping to the RKHS \mathrm{H}, k(.,)=$. $\langle\phi(),. \phi()$.$\rangle is the universal kernel associated with this$ mapping, and $N, M$ are the total number of items in the source and target respectively. In short, the MMD between the distributions of two datasets is equivalent to the distance between the sample means in a high-dimensional feature space.

\section{Experiments}

\subsection{Datasets}

We compare and evaluate our method against baseline approaches on a number of benchmark datasets so that fair comparisons can drawn. Such datasets are:

MNIST $\longrightarrow$ USPS (M $\rightarrow$ U): MNIST [23] is treated as source domain; USPS [9] is treated as target domain. As both these datasets contain grayscale images the domain 


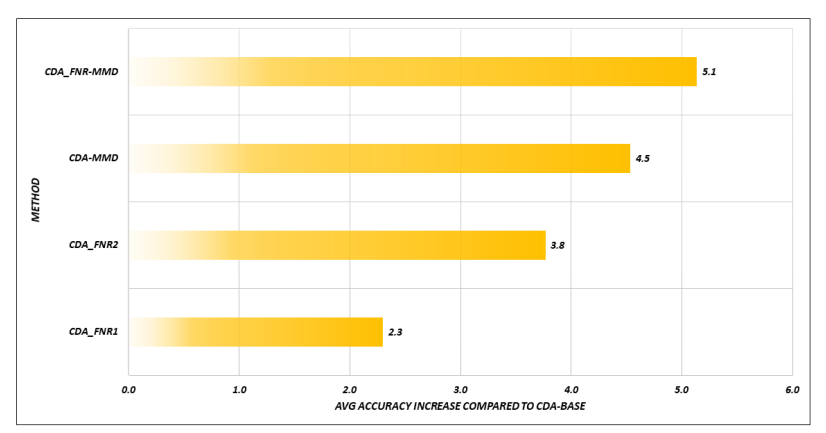

Figure 3: Average Accuracy comparision of proposed CDA frameworks with CDA-Base.

Figure 4: Sample images from datasets: MNIST-USPS

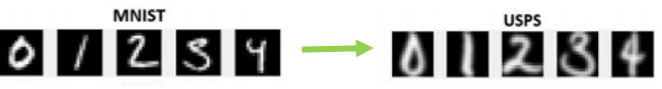

shift between these two datasets is relatively small. Figure4 shows sample images from $M \rightarrow U$.

SVHN $\longrightarrow$ MNIST $(M \rightarrow S)$ : In this setting, SVHN [30] is treated as source domain and MNIST is treated as the target domain. SVHN consists of crops of coloured streetview house numbers (in contrast to the grayscale images of MNIST), consisting of single digits extracted from images of urban house numbers from Google Street View. SVHN and MNIST are two digit classification datasets with a drastic distributional shift between the two of them. The adaptation from MNIST to SVHN is quite challenging because MNIST has a significantly lower intrinsic dimensionality than SVHN. Figure-5 shows sample images from $M \rightarrow S$.

Figure 5: Sample images from datasets: SVHN-MNIST

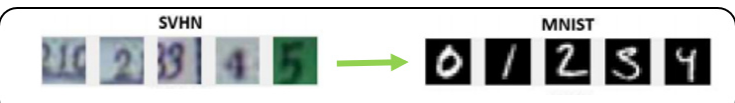

MNIST $\longrightarrow$ MNISTM $(\mathrm{M} \rightarrow \mathrm{MM}):$ MNIST [23] is treated as the source domain and MNISTM is treated as the target domain. MNISTM is a modification of MNIST dataset where the digits are blended with random patches from BSDS500 dataset color photos. Figure-6 shows sample images from $\mathrm{M} \rightarrow \mathrm{MM}$.

Figure 6: Sample images from datasets: MNIST-MNISTM

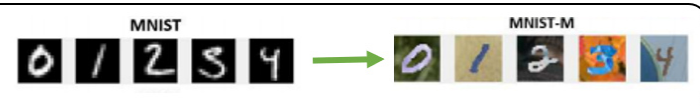

\subsection{Implementation Details}

CDA uses a base encoder ResNet-50 [14] trained from scratch followed by a two layered non-linear MLP. During pretraining, we train CDA on two Titan Xp GPUs, using LARS optimizer [44] with a batch size of 512 and weight decay of le- 6 for a total of 300 epochs. Similar to SimClr[4], we report performance by training a linear classifier on top of a fixed representation, but only with source labels to evaluate representations which is a standard benchmark that has been adopted by many papers in the literature $[4,5,31]$.

\subsection{Evaluation}

We conducted various experiments using unlabeled source and target digit datasets. As mentioned earlier, the goal of our experiments is to introduce contrastive learning to the domain adaptation problem in order to maximize generalization between source and target datasets by learning class discriminative and domain-invariant features along with improving the performance of contrastive loss by eliminating the false negatives. As shown below, We have performed multiple experiments using two views and four views [38]. Figure-3 compares the average accuracy of our proposed two-view CDA frameworks with CDA-Base. Following are the various experimental scenarios we considered on the digit datasets.

SimClr-Base: We start our experimental analysis by setting up SimClr. We have trained on source dataset using the same setup as SimClr, whilst testing on the target dataset. We treat this as a strong baseline which we call SimClrBase and use this as reference for comparison against other methods.

CDA-Base: We followed the methodology as described in section-3.2, trained the model based on equation-2 and evaluated on the target domain. Looking at table-1, we can clearly observe that the model demonstrates higher performance compared to the SimClr-Base. The difference in performanc can be attributed to the fact that the proposed model has clearly learnt both visual similarity and domaininvariance resulting in minimizing the distance between the domains and maximizing the classification accuracy. Overall, the average accuracy for all the datasets has increased by around $19 \%$ compared to the SimClr-Base model. We treat this result as a second strong baseline and call it CDA-Base.

CDA_FNR: We followed the methodology as described in section-3.3, and trained the model based on equation-4; the evaluation was done on the target domain dataset. Looking at table-1, in addition to learning visual similarity and domain-invariance, our model also successfully identified and eliminated the potential false negatives as they contain the same semantic information as that of the anchor, resulting in faster convergence and increased accuracy. We experimented on two scenarios; firstly we removed one false negative which we call FNR1 and in the second case, we 
experimented by removing two false negatives which we call FNR2. The results of these experiments can be seen in table-1, concluding that removal of false negatives improves accuracy and convergence time. The average accuracy has increased by $2.3 \%$ after removing one false negative. Additionally by removing two false negatives, we observe that the average accuracy has increased by $3.8 \%$ in comparison to CDA-Base and $1.5 \%$ in comparison to FNR1. Compared to the SimClr-Base, the average accuracy has increased around $21 \%$.

$C D A-M M D$ : We have used the same setup as that of CDA-Base. Additionally we introduced MMD as described in section 3.4, which is computed between vector representations extracted from each domain as per the equation-5, in order to reduce the distance between the source and target distributions. Finally, we backpropagate NT-Xent loss from equation-2 along with MMD loss equation-5. From table-2, we observe that by minimizing both these losses together, our model achieves much better alignment of the source and target domains, demonstrating the advantage of combined contrastive loss and MMD alignment. In comparison to the CDA-Base method, the performance gain tends to be comprehensive, as it has increased by $4.5 \%$.

CDA_FNR-MMD: We have used the same setup as that of CDA_FNR, and we have also introduced MMD, which is computed between vector representations extracted from each domain as per the equation-5, in order to reduce the distance between the source and target distributions. We calculate FNR loss both for source and target domains using equation-4 and backpropagate based on FNR and MMD loss functions. From table-2, we observe that by removing the potential false negatives and minimizing the discrepancy together, our model retains semantic information, hence converging faster and learning both visual similarity and domain-invariance; this is achieved by aligning source and target domains efficiently, showing the effectiveness of this method. In comparison to the CDA-Base method, the average performance gain tends to be larger as it has increased by a huge margin of $5.1 \%$.

Comparison with the state of art: Using our approach, we demonstrate that our model can perform very well in the domain adaptation setting without access to labeled data and imagenet parameters, just by training using the unlabeled data itself, in contrast to other unsupervised domain adaptation methods which have access to the source labels. We have compared our results with those of other state-of-the-art models, demonstrating that our model performs favorably in comparison with them. From table-3, we can conclude that our model has outperformed in the MNIST-USPS and SVHN-MNIST tasks compared to the other state-of-the-art models like DANN, DAN, ADDA, DDC and Simclr-Base [10, 28, 39, 27, 40, 4]

Inspired by [38], we have also performed similar experi-
Table 1: Accuracy values on the digits datasets evaluated using the proposed SimClr-Base and proposed CDA framework, along with the introduction of false negative removal. The best average is indicated in bold. M:MNIST, U:USPS, S:SVHN and MM:MNISTM.

\begin{tabular}{lcccc}
\hline Method & $\mathrm{M} \rightarrow \mathrm{U}$ & $\mathrm{S} \rightarrow \mathrm{M}$ & $\mathrm{M} \rightarrow \mathrm{MM}$ & $\mathrm{Avg}$ \\
\hline SimClr-Base [4] & 92.0 & 31.7 & 34.9 & 53.1 \\
CDA-Base & 92.5 & 64.8 & 57.9 & 71.7 \\
CDA_FNR1 & 93.2 & 69.4 & 59.5 & 74.0 \\
CDA_FNR2 & 94.1 & 71.7 & 60.6 & $\mathbf{7 5 . 5}$ \\
\hline
\end{tabular}

Table 2: Accuracy values on the digits datasets evaluated using CDA framework with the introduction of MMD and compared against base models. The best average is indicated in bold. M:MNIST, U:USPS, S:SVHN and MM:MNISTM.

\begin{tabular}{lcccc}
\hline Method & $\mathrm{M} \rightarrow \mathrm{U}$ & $\mathrm{S} \rightarrow \mathrm{M}$ & $\mathrm{M} \rightarrow \mathrm{MM}$ & Avg \\
\hline SimClr-Base [4] & 92.0 & 31.7 & 34.9 & 53.1 \\
CDA-Base & 92.5 & 64.8 & 57.9 & 71.7 \\
CDA-MMD & 93.4 & 74.8 & 60.6 & 76.2 \\
CDA_FNR-MMD & 94.2 & 76.2 & 60.2 & $\mathbf{7 6 . 8}$ \\
\hline
\end{tabular}

ments using four views on the digit datasets, which we compare with a) CDA-Base and b) Contrastive Domain Adaptation with Four Augmentations(CDAx4aug).

CDAx4aug: We have tested our method by using four augmentations per anchor per source and followed the methodology as described in section- 3.2 based on equation2. The only change is that we now backpropagate four contrastive losses two from the source and two from target domain. From table-4, we can observe that the additional augmentations have significantly improved the average accuracy of the method, compared to the two-view CDA-Base, due to the availability of additional positive and negative samples. Overall, by adding two additional views to the CDA-Base method we have gained an average accuracy of $5.1 \%$ compared to the CDA-Base method.

CDAx4aug_FNR: We followed the methodology as described in section-3.3, and trained the model based on equation-4 with four augmentations per domain as opposed to two. We then evaluated the trained model on the target domains. Looking at table-4, we can clearly establish that the additional views helped the model learn visual similarity and domain-invariance resulting in minimizing the distance between the domains. It also helped the model to successfully identify and eliminate the potential false negatives, thus resulting in converging faster along with an average accuracy increase of $5.8 \%$ compared to CDA-Base and $0.7 \%$ compared to CDAx4aug-Base.

CDAx4aug-MMD: We have used the same setup as that of CDAx4aug, but we also introduced MMD computed be- 
Table 3: Comparision of the proposed CDA method with state-of-the-art methods, using ACCURACY as the performance metric. The best numbers are indicated in bold. M:MNIST, U:USPS, S:SVHN and MM:MNISTM.

\begin{tabular}{lccc}
\hline Method & $\mathrm{M} \rightarrow \mathrm{S}$ & $\mathrm{S} \rightarrow \mathrm{M}$ & $\mathrm{M} \rightarrow \mathrm{MM}$ \\
\hline SimClr-Base [4] & 92.0 & 31.7 & 34.9 \\
DDC & 79.1 & 68.1 & - \\
ADDA & 89.4 & 76.0 & - \\
DANN & - & 73.8 & 76.6 \\
DAN & 81.1 & 71.1 & $\mathbf{7 6 . 9}$ \\
$\begin{array}{l}\text { CDA_FNR-MMD } \\
\text { (our method) }\end{array}$ & $\mathbf{9 4 . 2}$ & $\mathbf{7 6 . 2}$ & 60.2 \\
\hline
\end{tabular}

Table 4: Accuracy values on the digits datasets compared with Base models and evaluated using CDA framework with four views along with the introduction of false negative removal. The best average is indicated in bold. M:MNIST, U:USPS, S:SVHN and MM:MNISTM.

\begin{tabular}{lcccc}
\hline Method & $\mathrm{M} \rightarrow \mathrm{U}$ & $\mathrm{S} \rightarrow \mathrm{M}$ & $\mathrm{M} \rightarrow \mathrm{MM}$ & Avg \\
\hline SimClr-Base [4] & 92.0 & 31.7 & 34.9 & 53.1 \\
CDA-Base & 92.5 & 64.8 & 57.9 & 71.7 \\
CDAx4aug & 92.9 & 74.1 & 63.5 & 76.8 \\
CDAx4aug_FNR & 93.6 & 75.0 & 64.0 & $\mathbf{7 7 . 5}$ \\
\hline
\end{tabular}

Table 5: Accuracy values on the digits datasets evaluated using CDA framework with four views, along with the introduction of MMD compared with Base models. The best average is indicated in bold. M:MNIST, U:USPS, S:SVHN and MM:MNISTM.

\begin{tabular}{lcccc}
\hline Method & $\mathrm{M} \rightarrow \mathrm{S}$ & $\mathrm{S} \rightarrow \mathrm{M}$ & $\mathrm{M} \rightarrow \mathrm{MM}$ & Avg \\
\hline SimClr-Base [4] & 92.0 & 31.7 & 34.9 & 53.1 \\
CDA-Base & 92.5 & 64.8 & 57.9 & 71.7 \\
CDAx4aug & 92.9 & 74.1 & 63.5 & $\mathbf{7 6 . 8}$ \\
CDAx4aug-MMD & 92.7 & 69.3 & 58.6 & 73.5 \\
CDAx4aug & & & & \\
_FNR-MMD & 92.5 & 70.6 & 61.5 & 74.9 \\
\hline
\end{tabular}

tween vector representations extracted from each domain as per equation-5. We backpropagate XT-Xent loss for two pairs of source and two pairs of target domains, along with MMD loss. From table-5 we can observe that performance gain using MMD was not significant due to the noise from additional augmentations, resulting in slow convergence between the source and target distributions.

CDAx4aug_FNR-MMD: We have used the same setup as that of CDAx4aug_FNR, but we have additionally introduced MMD loss computed between vector representations extracted from each domain as per the equation-5, and backpropagated along with using FNR loss. From table5 , we can see that the average performance has increased compared to CDAx4aug-MMD, due to the false negative removal, but the addition of MMD has comparatively slowed convergence.

\section{Conclusion}

Over the past few years, ImageNet pre-training has become a de facto process in a model's development pipeline. In reality though, there exist various application areas, e.g. medical imaging, where getting labeled data is a very onerous, laborious and costly process. Improving upon methods and/or developing new ones that can extract knowledge from unlabeled data and then adapting them to a downstream task, will enable the adoption of machine learning algorithms at scale in application areas that have not seen much of a "machine learning revolution" yet, e.g. agri-food.

In this paper we demonstrated that via employing our proposed CDA approach and its variants we can train a model to perform competitively in a domain adaptation setting, without having access to labeled data or imagenet parameters, just by training using the unlabeled data itself. CDA also introduces identification and removal of the potential false negatives in the DA setting, resulting in improved accuracy. We also extend our framework to learn from more than two views in the DA setting and tested it using various experimental scenarios, demonstrating that it can be effectively used for downstream domain adaptation task. We hope that our work encourages future researchers to apply contrastive learning to domain adaptation.

\section{References}

[1] Bashar Alhnaity, Stefanos Kollias, Georgios Leontidis, Shouyong Jiang, Bert Schamp, and Simon Pearson. An autoencoder wavelet based deep neural network with attention mechanism for multistep prediction of plant growth. Information Sciences, 560, 2021. 1

[2] Francesco Caliva, Fabio Sousa De Ribeiro, Antonios Mylonakis, Christophe Demazi'ere, Paolo Vinai, Georgios Leontidis, and Stefanos Kollias. A deep learning approach to anomaly detection in nuclear reactors. In 2018 International joint conference on neural networks (IJCNN), pages 1-8. IEEE, 2018. 1

[3] Chao Chen, Zhihong Chen, Boyuan Jiang, and Xinyu Jin. Joint domain alignment and discriminative feature learning for unsupervised deep domain adaptation. In Proceedings of the AAAI conference on artificial intelligence, volume 33, pages 3296-3303, 2019. 2

[4] Ting Chen, Simon Kornblith, Mohammad Norouzi, and Geoffrey Hinton. A simple framework for contrastive learning of visual representations. arXiv preprint arXiv:2002.05709, 2020. 1, 2, 3, 4, 6, 7, 8

[5] Ting Chen, Simon Kornblith, Kevin Swersky, Mohammad Norouzi, and Geoffrey Hinton. Big self-supervised models are strong semi-supervised learners. arXiv preprint arXiv:2006.10029, 2020. 1, 2, 6 
[6] Zhihong Chen, Chao Chen, Xinyu Jin, Yifu Liu, and Zhaowei Cheng. Deep joint two-stream wasserstein autoencoder and selective attention alignment for unsupervised domain adaptation. Neural computing and applications, pages 1-14, 2019. 2

[7] Ching-Yao Chuang, Joshua Robinson, Lin Yen-Chen, Antonio Torralba, and Stefanie Jegelka. Debiased contrastive learning. arXiv preprint arXiv:2007.00224, 2020. 2, 3

[8] Fabio De Sousa Ribeiro, Georgios Leontidis, and Stefanos Kollias. Introducing routing uncertainty in capsule networks. In H. Larochelle, M. Ranzato, R. Hadsell, M. F. Balcan, and H. Lin, editors, Advances in Neural Information Processing Systems, volume 33, pages 6490-6502. Curran Associates, Inc., 2020. 1

[9] John S Denker, WR Gardner, Hans Peter Graf, Donnie Henderson, Richard E Howard, W Hubbard, Lawrence D Jackel, Henry S Baird, and Isabelle Guyon. Neural network recognizer for hand-written zip code digits. In Advances in neural information processing systems, pages 323-331. Citeseer, 1989. 5

[10] Yaroslav Ganin, Evgeniya Ustinova, Hana Ajakan, Pascal Germain, Hugo Larochelle, François Laviolette, Mario Marchand, and Victor Lempitsky. Domain-adversarial training of neural networks. The journal of machine learning research, 17(1):2096-2030, 2016. 2, 7

[11] Swarnendu Ghosh, Anisha Pal, Shourya Jaiswal, KC Santosh, Nibaran Das, and Mita Nasipuri. Segfast-v2: Semantic image segmentation with less parameters in deep learning for autonomous driving. International Journal of Machine Learning and Cybernetics, 10(11):3145-3154, 2019. 1

[12] Arthur Gretton, Karsten M Borgwardt, Malte J Rasch, Bernhard Schölkopf, and Alexander Smola. A kernel two-sample test. Journal of Machine Learning Research, 13(Mar):723773, 2012. 2, 5

[13] Kaiming He, Haoqi Fan, Yuxin Wu, Saining Xie, and Ross Girshick. Momentum contrast for unsupervised visual representation learning. In Proceedings of the IEEE/CVF Conference on Computer Vision and Pattern Recognition, pages 9729-9738, 2020. 1, 2

[14] Kaiming He, Xiangyu Zhang, Shaoqing Ren, and Jian Sun. Deep residual learning for image recognition. In Proceedings of the IEEE conference on computer vision and pattern recognition, pages 770-778, 2016. 4, 6

[15] Miao He and David He. A new hybrid deep signal processing approach for bearing fault diagnosis using vibration signals. Neurocomputing, 396:542-555, 2020. 1

[16] Tri Huynh, Simon Kornblith, Matthew R Walter, Michael Maire, and Maryam Khademi. Boosting contrastive selfsupervised learning with false negative cancellation. arXiv preprint arXiv:2011.11765, 2020. 1, 3

[17] Yannis Kalantidis, Mert Bulent Sariyildiz, Noe Pion, Philippe Weinzaepfel, and Diane Larlus. Hard negative mixing for contrastive learning. arXiv preprint arXiv:2010.01028, 2020. 1, 3

[18] Guoliang Kang, Lu Jiang, Yi Yang, and Alexander G Hauptmann. Contrastive adaptation network for unsupervised domain adaptation. In Proceedings of the IEEE/CVF Confer- ence on Computer Vision and Pattern Recognition, pages 4893-4902, 2019. 2

[19] Stefanos Karakanis and Georgios Leontidis. Lightweight deep learning models for detecting covid-19 from chest x-ray images. Computers in Biology and Medicine, 130:104181. 1

[20] Prince Waqas Khan, Yung-Cheol Byun, and Namje Park. Iot-blockchain enabled optimized provenance system for food industry 4.0 using advanced deep learning. Sensors, 20(10):2990, 2020. 1

[21] Donghyun Kim, Kuniaki Saito, Tae-Hyun Oh, Bryan A Plummer, Stan Sclaroff, and Kate Saenko. Cross-domain self-supervised learning for domain adaptation with few source labels. arXiv preprint arXiv:2003.08264, 2020. 2

[22] Yann LeCun, Yoshua Bengio, and Geoffrey Hinton. Deep learning. nature, 521(7553):436-444, 2015. 1

[23] Yann LeCun, Léon Bottou, Yoshua Bengio, and Patrick Haffner. Gradient-based learning applied to document recognition. Proceedings of the IEEE, 86(11):2278-2324, 1998. 5,6

[24] Chen-Yu Lee, Tanmay Batra, Mohammad Haris Baig, and Daniel Ulbricht. Sliced wasserstein discrepancy for unsupervised domain adaptation. In Proceedings of the IEEE/CVF Conference on Computer Vision and Pattern Recognition, pages 10285-10295, 2019. 2

[25] Yi Li, Huahong Zhang, Camilo Bermudez, Yifan Chen, Bennett A Landman, and Yevgeniy Vorobeychik. Anatomical context protects deep learning from adversarial perturbations in medical imaging. Neurocomputing, 379:370-378, 2020. 1

[26] Dongfang Liu, Yiming Cui, Yingjie Chen, Jiyong Zhang, and Bin Fan. Video object detection for autonomous driving: Motion-aid feature calibration. Neurocomputing, 409:1-11, 2020. 1

[27] Ming-Yu Liu and Oncel Tuzel. Coupled generative adversarial networks. arXiv preprint arXiv:1606.07536, 2016. 7

[28] Mingsheng Long, Yue Cao, Jianmin Wang, and Michael Jordan. Learning transferable features with deep adaptation networks. In International conference on machine learning, pages 97-105. PMLR, 2015. 2, 7

[29] Mingsheng Long, Han Zhu, Jianmin Wang, and Michael I Jordan. Deep transfer learning with joint adaptation networks. In International conference on machine learning, pages 2208-2217. PMLR, 2017. 2

[30] Yuval Netzer, Tao Wang, Adam Coates, Alessandro Bissacco, Bo Wu, and Andrew Y Ng. Reading digits in natural images with unsupervised feature learning. 2011. 6

[31] Aaron van den Oord, Yazhe Li, and Oriol Vinyals. Representation learning with contrastive predictive coding. arXiv preprint arXiv:1807.03748, 2018. 6

[32] Changhwa Park, Jonghyun Lee, Jaeyoon Yoo, Minhoe Hur, and Sungroh Yoon. Joint contrastive learning for unsupervised domain adaptation. arXiv preprint arXiv:2006.10297, 2020. 2

[33] Hendrik Purwins, Bo Li, Tuomas Virtanen, Jan Schlüter, Shuo-Yiin Chang, and Tara Sainath. Deep learning for audio signal processing. IEEE Journal of Selected Topics in Signal Processing, 13(2):206-219, 2019. 1 
[34] Fabio De Sousa Ribeiro, Francesco Calivá, Mark Swainson, Kjartan Gudmundsson, Georgios Leontidis, and Stefanos Kollias. Deep bayesian self-training. Neural Computing and Applications, pages 1-17, 2019. 1

[35] Joshua Robinson, Ching-Yao Chuang, Suvrit Sra, and Stefanie Jegelka. Contrastive learning with hard negative samples. arXiv preprint arXiv:2010.04592, 2020. 1, 3

[36] Baochen Sun and Kate Saenko. Deep coral: Correlation alignment for deep domain adaptation. In European conference on computer vision, pages 443-450. Springer, 2016. 2

[37] Mamatha Thota, Stefanos Kollias, Mark Swainson, and Georgios Leontidis. Multi-source domain adaptation for quality control in retail food packaging. Computers in Industry, 123:103293, 2020. 1

[38] Yonglong Tian, Dilip Krishnan, and Phillip Isola. Contrastive multiview coding. arXiv preprint arXiv:1906.05849, 2019. 2, 6, 7

[39] Eric Tzeng, Judy Hoffman, Kate Saenko, and Trevor Darrell. Adversarial discriminative domain adaptation. In Proceedings of the IEEE conference on computer vision and pattern recognition, pages 7167-7176, 2017. 2, 7

[40] Eric Tzeng, Judy Hoffman, Ning Zhang, Kate Saenko, and Trevor Darrell. Deep domain confusion: Maximizing for domain invariance. arXiv preprint arXiv:1412.3474, 2014. 2,7

[41] Dequan Wang, Coline Devin, Qi-Zhi Cai, Fisher Yu, and Trevor Darrell. Deep object-centric policies for autonomous driving. In 2019 International Conference on Robotics and Automation (ICRA), pages 8853-8859. IEEE, 2019. 1

[42] Xiongwei Wu, Doyen Sahoo, and Steven CH Hoi. Recent advances in deep learning for object detection. Neurocomputing, 396:39-64, 2020. 1

[43] Zhirong Wu, Yuanjun Xiong, Stella X Yu, and Dahua Lin. Unsupervised feature learning via non-parametric instance discrimination. In Proceedings of the IEEE Conference on Computer Vision and Pattern Recognition, pages 37333742, 2018. 2

[44] Yang You, Igor Gitman, and Boris Ginsburg. Large batch training of convolutional networks. arXiv preprint arXiv:1708.03888, 2017. 6 